\title{
REALISMO PRAGMÁTICO, RELATIVIDAD CONCEPTUAL Y VERDAD ABSOLUTA*
}

LEÓN OLIVÉ

InstTtuto de InVESTigaciones Fulosóficas

UNAM

En este trabajo sugeriré una forma de desarrollar las ideas del realismo interno - propuesto por Hilary Putnam, y llamado más recientemente realismo pragmático- de manera que resulten compatibles con una concepción de la verdad como adecuación y como aceptabilidad racional, la cual resultará ser absolutista pero a la vez compatible con una concepción ontológica relativista (véanse Putnam, 1981, 1987 y 1990).

Sostendré que, si es correcto el punto de vista internalista, según el cual los hechos y los objetos no son independientes con respecto a los marcos conceptuales, entonces la existencia de lo que llamamos hechos está ligada con las buenas razones para creer en ellos, dentro de los mismos marcos conceptuales. Esto es lo que justifica, en mi opinión, la adhesión a una concepción epistémica de la verdad. Así, defenderé la idea de que la verdad, además de adecuación entre el entendimiento y la realidad, involucra la aceptabilidad racional en condiciones epistémicas y de diálogo óptimas.

En consecuencia sostendré que es posible mantener una posición realista con respecto al conocimiento, un relativismo moderado que niega la inconmensurabilidad absoluta de los marcos conceptuales, pero que acepta que la existencia de hechos y de objetos es relativa a los marcos conceptuales, todo esto, junto con una idea absolutista de la verdad, a la cual se le concibe como una propiedad de las proposiciones que debe preservarse a través de los cambios de marcos conceptuales.

- Este artículo presenta de una manera ligeramente modificada la ponencia que se leyó en el XI Simposio de Filosofía del Instituto de Investigaciones Filosóficas de la UNAM, celebrado en Taxco, Guerrero, en torno a la obra de Hilary Purnam, en agosto de 1992. Las ideas centrales sobre el tipo de realismo intemo y sobre la noción de verdad que aquí se defienden, también las he sostenido en mi contribución al volumen del homenaje a Luis Villoro, compilado por Ernesto Garzón Valdés y Fernando Salmerón, el cual será editado por el Instituto de Investigaciones Filosóficas. Mientras el trabajo que aquí se presenta contiene algunas precisiones que considero importantes, aquél desarrolla más el contraste y precisa más a fondo las coincidencias y diferencias entre la posición de Villoro y la que por mi parte deseo defender. 
Veamos las tesis centrales del tipo de realismo que deseo defender:

a) Los hechos dependen de los marcos conceptuales (aunque también de la realidad independiente de los marcos).

b) La verdad es adecuación entre el entendimiento y la realidad.

Por marco conceptual entenderé el conjunto de creencias y saberes, de presupuestos metafísicos, de normas y valores, así como de reglas de inferencia y reglas metodológicas que los seres humanos presuponen y aplican en las interacciones con el mundo mediante las cuales pretenden obtener conocimiento de ese mundo.

Ahora bien, de acuerdo con la tesis a), la realidad mencionada en la tesis b), con la que existe adecuación, es una realidad constituida por hechos y por objetos que dependen (incluso en su existencia) de los marcos conceptuales. Por consiguiente, si se acepta la tesis a), es sostenible la tesis:

c) La verdad también significa aceptabilidad racional, en condiciones epistémicas y de diálogo óptimas.

Las condiciones epistémicas óptimas las entiendo a la manera de Putnam en el prefacio a Realism with a Human Face: "Si digo thay una silla en mi estudio', una situación epistémica ideal sería la de estar en mi estudio con las luces encendidas o con la luz del día pasando a través de la ventana, con ningún problema con mi vista, con una mente que no esté confundida, sin haber tomado drogas o estado sujeto a hipnósis, etc., y mirar y ver si hay una silla ahi”" (p. viii). Por condiciones de diálogo óptimas, quiero decir las condiciones de las que Habermas ha hablado ampliamente: aquellas en las que el interés prevaleciente es el de llegar a un consenso en relación con la verdad de las proposiciones y en donde no hay ningún ejercicio de poder que no sea el de la fuerza del argumento.

De manera más precisa, la tesis b) quiere decir que si ' $p$ ' es verdadera, es un hecho que $p$. Y la tesis c) significa que si ' $p$ ' es verdadera, en el marco conceptual en relación con el cual se afirma ' $p$ ' y ' $p$ ' tiene sentido, existen buenas razones a favor de ' $p$ ', y no pueden existir buenas razones en contra de la aceptación de ' $p$ '.

Más aún, si es correcta la tesis a), es decir la idea de que los hechos y los objetos reales dependen de los marcos conceptuales, entonces la verdad no puede interpretarse como adecuación entre el entendimiento y una realidad descontaminada de conceptos, sino que debe entenderse como adecuación entre el entendimiento y la realidad construida mediante la aplicación de marcos conceptuales.

Una consecuencia de esto es que, si los hechos son construidos a partir de la realidad independiente de los marcos conceptuales $y$ a partir de ca- 
da marco conceptual, lo que puede ser un hecho desde la perspectiva de un cierto marco conceptual, puede no serlo desde la perspectiva de otro, lo cual parece conducir a un relativismo fuerte con respecto a la verdad.

Éste es un tipo de relativismo que deseo evitar, para ello debemos precisar la tesis b) en el siguiente sentido:

$\left.b^{\prime}\right)$ si ' $p$ ' es verdadera, $p$ es un hecho desde la perspectiva de todo marco conceptual en donde ' $p$ ' tenga sentido y preserve su significado.

Pero si $p$ es un hecho desde la perspectiva de todo marco conceptual en donde ' $p$ ' tenga sentido, entonces dentro de cada uno de esos marcos conceptuales existirán razones a favor de la aceptación de 'p', y no habrá razones suficientes para el rechazo de ' $p$ ', en condiciones epistémicas y de diálogo óptimas.

Por consiguiente, si aceptamos las tesis a) y $\mathrm{b}^{\prime}$ ), también podemos sostener la tesis:

$\left.c^{\prime}\right)$ si ' $p$ ' es verdadera, desde la perspectiva de todo marco conceptual en donde ' $p$ ' tenga sentido y preserve su significado, ' $p$ ' es aceptable en condiciones epistémicas y de diálogo óptimas, esto es, pueden darse buenas razones a su favor, y no pueden existir buenas razones —en cada uno de esos marcos- en contra de la aceptación de ' $p$ '.

Esto no significa que de hecho ' $p$ ' sea aceptada por todos los sujetos epistémicos pertinentes. Significa que en condiciones epistémicas y de diálogo óptimas, los sujetos epistémicos pertinentes aceptarían ' $p$ '.

En lo que sigue trataré de fundamentar la adhesión a las tesis a), b') y $c^{\prime}$ ). Veremos que estas tres tesis no significan que no tengamos acceso epistémico al mundo real, sino que lo que conocemos es parte del mundo real, pero siempre "recortado" de acuerdo con un cierto marco conceptual y sus herramientas.

\section{La concepción del realismo}

Considero correcta una concepción muy general del realismo tal que, como la ha subrayado Luis Villoro, señale que "todo realismo admite la existencia independiente del mundo" (Villoro, 1990, p. 77).

Un problema central, sin embargo, es la concepción de "mundo" que se asocie con esta idea. Por mi parte quiero defender una concepción internalista, según la cual, en la medida en la que no es correcto concebir la existencia de los hechos y de los objetos como teniendo determinaciones intrínsecas, es decir determinaciones que existen con independencia del contexto en el que se encuentren y de sus relaciones con otros objetos y hechos, entonces la existencia misma de los hechos y de los objetos depende del contexto en el que se encuentren; pero los contextos, a la vez, nunca son absolutos, ni tiene 
sentido hablar de un contexto en sí mismo. Los contextos siempre son partes del mundo recortadas de acuerdo con los recursos conceptuales e intereses de los sujetos cognoscentes. Por esto, los hechos y objetos, al depender de los contextos, también dependen de los marcos conceptuales que posibilitan el recorte de los contextos relevantes, así como el enunciado de la existencia de los hechos o de los objetos. El mundo, entendido como la totalidad de hechos y de objetos, bajo esta perspectiva, no es independiente del marco conceptual a disposición de los sujetos cognoscentes. Como sugeriré adelante, ésta sigue siendo una concepción realista porque esa dependencia de los hechos y de los objetos con respecto de los marcos conceptuales no hace que los hechos o los objetos sean menos reales. Desde este punto de vista, las proposiciones verdaderas enuncian hechos, y esos hechos realmente existen.

Esta posición sostiene que efectivamente existe una realidad independiente de los marcos conceptuales, pero esa realidad no está conformada y dividida "naturalmente" en los hechos y los objetos que conocen los seres humanos. Esa realidad no necesariamente está dividida en sí misma en hechos y objetos. Desde la concepción que quiero defender, en el sentido del realismo interno de Putnam, el mundo de hechos y objetos es una construcción que se hace a partir de la realidad independiente, por medio de la intervención de los marcos conceptuales. Pero no por eso los hechos y los objetos no son reales, pues se construyen también a partir de la realidad que es independiente de los marcos conceptuales. Lo que ocurre es que esta última no está dividida, previamente a la aplicación de los marcos conceptuales en el proceso de generación y aceptación del conocimiento, en los hechos y objetos que conocemos. Es en el proceso de esa aplicación de los marcos conceptuales que nosotros establecemos los contextos relevantes y así dividimos la realidad en los objetos y hechos que conocemos. En este sentido puede decirse que es mediante la aplicación de los marcos que esos hechos y objetos vienen a la existencia. No se trata de hechos y objetos que existan previamente a la aplicación de los marcos que recortan la realidad independiente, es decir, que intervienen en la conformación de los contextos en los que tiene sentido hablar de la existencia de hechos y de objetos.

Es necesario distinguir entre la realidad constituida por los hechos y los objetos, y la realidad independiente de los marcos conceptuales. El realismo interno sostiene que la realidad de hechos y objetos no es independiente de los marcos conceptuales, ni en un sentido ontológico. La llamaré la realidad $\mathrm{d}_{\mathrm{c}}$. Pero no es que se trate de la realidad de los hechos concebidos, conocidos o juzgados por los seres humanos, se trata de la realidad de hechos y de objetos en la que se divide el mundo al aplicar un cierto marco conceptual con el fin de conocer la realidad independiente, sin importar que los hechos u objetos sean o no de becho conocidos, concebidos o juzgados. La realidad $\mathrm{c}_{\mathrm{c}}$ de hechos y de objetos depende noseológicamente y ontológicamente de los marcos conceptuales. 
Pero desde un punto de vista que pretende seguir siendo realista, es necesario aceptar la realidad independiente de los marcos conceptuales, como la realidad a partir de la cual se construyen los hechos y los objetos mediante la aplicación de los marcos conceptuales. Desde el punto de vista internalista, no tenemos ninguna razón para pensar que esta realidad está dividida previamente a la aplicación de los marcos conceptuales en hechos y objetos, pero en cambio sí se concibe como independiente de los marcos conceptuales.

Sugiero que como tesis metafísica —defendible por argumento filosófico a partir de las condiciones que la realidad debe cumplir para que seres racionales como nosotros la podamos conocer- lo más que podemos sostener es que esa realidad independiente es compleja, y que tiene una estructura compleja, la cual sin embargo no podemos aprehender como una totalidad, sino que la aprehendemos siempre en relación con contextos que se demarcan mediante la aplicación de los marcos conceptuales.

Estoy consciente de que se requeriría una larga elaboración y argumentación en torno a esta concepción metafísica. Por ahora sólo la dejaré apuntada, y sugeriré la manera en la que puede verse la relación de la realidad ${ }_{c}$ de los hechos y objetos constituidos por los marcos conceptuales y la realidad independiente.

La formulación misma de la concepción sugiere la relación de la que se trata. No es que sean dos realidades o dos mundos distintos entre los cuales, por ejemplo, hubiera correspondencia. Se trata más bien de la relación entre un subconjunto y un conjunto que lo contiene. La realidad de hechos y de objetos, constituidos a partir de los marcos conceptuales, es parte de la realidad independiente, la cual tiene una existencia previa a la de los marcos conceptuales. La realidad independiente es recortada en un mundo de hechos y objetos mediante la aplicación de cada marco conceptual. Por eso los hechos y objetos son aspectos de la realidad independiente, pero en tanto que hechos y objetos no tienen una existencia absoluta, no son independientes del marco conceptual por medio del cual la realidad independiente fue recortada.

$S i$ pensamos que la realidad ${ }_{c}$ está formada por hechos y por objetos, que existen por la aplicación de los marcos conceptuales, entonces esa realidad ${ }_{c}$ no es independiente (ni ontológicamente) de los marcos conceptuales.

Veamos con mayor detalle qué es lo que desde esta interpretación se entiende como hecho y como objeto, y por qué los hechos y los objetos dependen ontológicamente de los marcos conceptuales.

Un hecho es una situación en el mundo que realmente ha ocurrido, está ocurriendo o va ocurrir. Igualmente, un objeto es una entidad que realmente existe en el mundo, ha existido o existirá. Pero no habría ningún hecho, ni objeto alguno, si no fuera por la aplicación de algún marco conceptual a la realidad. Al aplicarse un marco conceptual a la realidad se establece el contexto relevante con respecto al cual se deberán identificar las propiedades del hecho 
o del objeto que determinan su identidad en tanto que ese objeto o ese hecho. Es en este sentido que los hechos y los objetos se crean, vienen a la existencia. Si no se da la acción de recortar a la realidad independiente en contextos relevantes, no existen los hechos ni los objetos.

¿Sigue siendo esta posición una posición realista? Sostengo que sí, porque los hechos y los objetos que se constituyen al aplicarse los marcos conceptuales son reales, porque son aspectos de la realidad independiente. El mundo (de los hechos y los objetos) depende noseológicamente de los marcos conceptuales. No hay objetos sin conceptos; en tanto que todos los objetos, para ser identificables desde algún marco conceptual, requieren de su correspondiente concepto - aunque éste pueda no estar definido con precisión. Pero desde el punto de vista internalista incluso los hechos y los objetos dependen en su existencia de los marcos conceptuales. Esto significa que todo objeto, para ser tal, existe dentro de un cierto contexto, en el cual se establecen sus propiedades definitorias, y este contexto, a su vez, debe haber sido determinado a partir de algún marco conceptual. Por esto, se puede decir que sin marco conceptual el objeto no existiría.

Cuando yo digo, "la montaña que está frente a mí tiene millones de años y por consiguiente ha existido mucho antes de que existiera la posibilidad en la Tierra de que fuera percibida y concebida como objeto por seres racionales", tengo razón. Pero el objeto que denomino con el término 'montaña' es sólo un aspecto de la realidad que ofrece su presencia y que soporta la existencia del objeto constituido, percibido y concebido por los seres humanos, a saber, el que nosotros denominamos montaña.

La idea es que hay una realidad compleja, a partir de la cual se "recorta" el contexto en el que se dan las propiedades que determinan eso que llamamos montaña y que hacen que ese objeto sea una montaña. Pero la montaña es un aspecto de esa realidad, por eso realmente existe. Pero no es un aspecto absoluto de la realidad, cuyas propiedades definitorias queden establecidas intrínsecamente para el objeto montaña como si pudiera tener una existencia absoluta y aislada de cualquier otro objeto en la realidad. Por esto, la existencia de la montaña no es independiente del hecho de que los seres humanos intervengan con sus marcos conceptuales para recortar una fracción de la realidad en donde quedan determinados los objetos que pueden ser percibidos por ellos en función de la forma en la que están constituidos biológica y cognitivamente, de sus intereses, y de los recursos de sus marcos conceptuales.

Los objetos así constituidos existen realmente, no es que sólo existan como objetos pensados. La montaña existe como montaña por la acción y el pensamiento de los seres humanos. Pero no es que la montaña sea un mero objeto pensado y que no sea una montaña real. La montaña real es un aspecto de la realidad independiente de los seres humanos y sus marcos conceptuales, pero es un objeto que sólo existe en relación con los marcos conceptuales. 
A partir de esa realidad independiente, y mediante la aplicación de los marcos conceptuales, es que se ha constituido el objeto montaña, el cual, entre otras características tiene la edad de millones de años y, por consiguiente, es anterior a la aparición de marcos conceptuales sobre la Tierra. La edad de un objeto natural, como la montaña, es la edad de un aspecto de la realidad independiente. Por eso no hay nada de raro en afirmar que la montaña es más vieja que, digamos, la especie bomo sapiens.

Sobre esa realidad independiente, como totalidad, podemos decir pocas cosas, y lo que digamos por supuesto no tendrá el carácter de conocimiento empírico. El conocimiento empírico lo es sólo de aspectos de la realidad independiente, de hechos y de objetos, no de esa realidad como totalidad. Lo que se diga de la realidad independiente, como totalidad, tendrá el carácter de tesis metafísicas, defendibles por argumentación filosófica. Así, no sabemos cómo es ni qué es esa realidad independiente. Con respecto a la montaña podría ser la Tierra entera o el Universo entero. Pero como quiera que sea esa realidad, la montaña es un objeto construido por los seres humanos, constituido por aspectos de la realidad, los cuales son destacados por los seres humanos de acuerdo con sus intereses, sus capacidades cognitivas y los recursos de sus marcos conceptuales.

Pero no se trata de que la montaña no sea montaña más que si la nombramos "montaña"; es decir, no se trata de una mera cuestión lingüística. Se trata de que la montaña no es montaña a menos que se haga un recorte de la realidad independiente, se establezca así un cierto contexto y dentro de él se constituyan los objetos y hechos relativos a ese contexto, en relación con los cuales se constituye el objeto que es la montaña.

El realismo interno sostiene que existe una realidad independiente de los seres humanos, de sus marcos conceptuales y de sus recursos para conocer el mundo.

Tratando de precisar la idea del realismo interno, podemos afirmar lo siguiente: los seres humanos demarcan contextos y así dividen a la realidad en piedras, árboles y montañas, de acuerdo con lo que pueden percibir, concebir y manipular, y de acuerdo con lo que a ellos les interesa. El objeto montaña está conformado por otros objetos, tales como piedras, porque los seres humanos pueden recortar visualmente la montaña en piedras, pueden desgajar partes de esa montaña rocosa en piedras que pueden ver, tocar y manipular, arrojándolas como armas, recortándolas para construir casas, etc. Pero la piedra que percibo frente a mí, aunque nunca haya sido tocada por un ser humano, es una piedra porque así se percibe desde el punto de vista de los seres humanos y así se concibe desde sus marcos conceptuales. Igualmente esa piedra puede ser parte de la montaña, pero verla como parte de la montaña es también verla como un objeto constituido como tal, como objeto, 
por intervención de los seres humanos. Es interesante imaginar cómo constituiríamos a la montańa y a las piedras como objetos si tuvieramos el tamaño de una amiba, o si fuéramos gigantes de un kilómetro de altura. Lo mismo ocurre con otros objetos tales como átomos, electrones y neutrinos. También pueden formar parte de los objetos que conforman la montaña. Pero al mismo tiempo esos objetos han sido constituidos como tales porque algunos de los marcos conceptuales de los seres humanos han contado con los recursos teóricos para identificar las propiedades que determinan esos objetos dentro del contexto pertinente, y también ha interesado a algunos seres humanos hacer ese tipo de "recorte" de la realidad, estableciendo el contexto relevante y, ya en él, identificando al objeto - con el interés, entre otros, de manipular la realidad, aunque los átomos sean manipulados de manera bastante diferente a como se manipulan las piedras.

Así, la noción de 'mundo real' debe verse como no independiente del marco conceptual, en el sentido de que ese mundo real, la realidad ${ }^{\text {, depende del }}$ marco conceptual, no únicamente en el sentido noseológico de que depende en tanto que mundo conocido, concebido y juzgado por los miembros de la comunidad epistémica que comparten ese marco conceptual, sino en el sentido más fuerte de mundo de objetos y de hechos cuya existencia depende de ese mismo marco conceptual. Pero esto es compatible con el reconocimiento de una realidad independiente de todo marco conceptual, la cual es compleja y no está dividida por sí misma en hechos y objetos. Como hemos visto, los hechos y los objetos existen mediante la confluencia de esa realidad independiente y los marcos conceptuales.

\section{Verdad}

Siguiendo la anterior interpretación del realismo interno, podemos pasar a la discusión de la noción verdad.

En primer lugar, quisiera señalar que, como lo ha subrayado Villoro en el contexto de una discusión reciente acerca de estos mismos problemas, podemos seguir considerando que un hecho es lo que hace verdadera a una proposición; algo que está allí, en el mundo real y que es aseverado. Un hecho es una parte de la realidad que no es puesto ahí por mi acto de enunciarlo (al hecho) (Villoro 1990, p. 84). Pero Villoro dice que seremos capaces de juzgar como real el hecho que ha sido percibido o enunciado, en la medida en la que podamos demostrar que el hecho está dado y no propuesto, en la medida en la que se me imponga y no sea un resultado de mis deseos (ibid.). "Pero al inferir la realidad del hecho, simplemente afirmo que no ha sido fraguado por mí, sino que forma parte de esa esfera de lo impuesto a mis acciones, deseos y creencias. Lo que asevero es que en el hecho confluyen ("se corresponden") lo aseverado y lo presente o impuesto." (Ibid.) 
Me gustaría ir más allá de lo que dice Villoro y afirmar - lo que entiendo que sería el núcleo de un realismo interno- que el hecho es esa confluencia de lo que es enunciado y de lo que es impuesto. Lo que es enunciado incluye lo que el sujeto pone, y lo que está presente o es impuesto es lo que es dado por la realidad independiente.

Pero no considero que sea correcto suponer que un hecho (en singular) está dado o es impuesto por la realidad independiente. Lo que es independiente de mis deseos y creencias es la realidad independiente en cuanto tal, en su totalidad. Toda ella es la que me ofrece resistencia, y es independiente de mis deseos y de mi voluntad. Pero cuando confronto esa realidad con mi marco de intereses, conocimientos, creencias y otras condiciones subjetivas e intersubjetivas, y entonces ocurre la confluencia de la realidad independiente con mi marco conceptual, entonces vienen los hechos a la existencia.

Bajo esta perspectiva, sugiero analizar la noción de verdad de la siguiente manera: Cuando digo "es verdad que $p$ ", no creo que sea lo más adecuado interpretar que lo que pretendo es decir que $p$ se me impone o que me sale al encucntro, sino que pienso que se debe interpretar como diciendo que la realidad independiente se me resiste y me sale al encuentro, y que de acuerdo con mis intereses, mi aparato perceptual, mis condiciones subjetivas y mi marco de actitudes y creencias - mi marco conceptual - yo recorto la realidad de tal manera que dentro del contexto relevante -el contexto de la realidad que mi marco ha recortado- $p$ es un hecho, es decir $p$ existe. Pero entonces su existencia no es independiente de mi marco conceptual, como tampoco lo es de la realidad independiente.

Por supuesto la pretensión de la que se habla arriba no es una pretensión de la que tenga que estar consciente un sujeto epistémico al sostener la verdad de una proposición ' $p$ '. La idea es más bien que al sostener la verdad de ' $p$ ', un sujeto epistémico pretende que $p$ es. Pero lo que he sugerido es que según la perspectiva del realismo interno, pretender que $p$ es el caso significa que $p$ queda determinado dentro del contexto que mi marco conceptual me ha permitido recortar en la realidad independiente, sin que el sujeto tenga que estar consciente de esto.

Pero entonces, aunque es correcto decir que podemos juzgar como real un hecho cuando nos percatamos y tenemos razones para creer que no se debe a ilusión o a engaño, no es exacto decir que "podremos juzgar como 'real' al hecho percibido o enunciado en la medida en que comprobemos que efectivamente está dado y no propuesto, que efectivamente se me impone y no es un producto más de mi deseo" (Villoro, loc. cit.); pues de acuerdo con el realismo pragmático, los hechos no están dados, aunque tampoco son productos de nuestros deseos, ni están fraguados por nosotros ni son propuestos por nosotros. Los hechos se producen porque confluye la realidad independiente 
y los marcos conceptuales de los sujetos. Pero ni siquiera los marcos conceptuales, y mucho menos la realidad independiente, dependen de la voluntad o de los deseos de los sujetos.

Esta situación es la que permite admitir una concepción de la verdad como adecuación, por un lado, y como aceptabilidad en condiciones epistémicas y de diálogo óptimas, por el otro. Por una parte, si ' $p$ ' es verdadera, $p$ es un hecho, una parte de la realidad. $Y$ es correcto decir que el hecho hace verdadera la proposición.

Pero si además admitimos que los hechos no son independientes de los marcos conceptuales, más aún, que los marcos conceptuales son determinantes en la constitución de los hechos, entonces el que $p$ sea un hecho no es independiente de las buenas razones que existan en el marco conceptual pertinente para la aceptación de ' $p$ '.

Así, la idea es que si ' $p$ ' es verdadera, entonces hay una doble implicación: que

i) $p$ es un hecho,

y puesto que $p$ es un hecho, $p$ es construido a partir de mi marco conceptual, por consiguiente:

ii) dentro de mi marco es posible aducir razones a favor de ' $p$ ' y no pueden existir buenas razones para dudar que $p$ sea un hecho.

Así, si ' $p$ ' es verdadera, $p$ es un hecho, independientemente de si otros sujetos epistémicos pertinentes admiten que sea un hecho. Pero el que $p$ sea un hecho no es independiente de que existan buenas razones para aceptar o rechazar - racionalmente, bajo condiciones epistémicas y de diálogo óptimasque $p$ es un hecho.

Más aún, si ' $p$ ' es verdadera, y por consiguiente $p$ es un hecho, esto significa que ningún sujeto, cualquiera que sea su marco conceptual -siempre y cuando en su marco conceptual ' $p$ ' tenga sentido y preserve su significado- podrá sostener, en condiciones óptimas de discusión y de diálogo, buenas razones para rechazar ' $p$ ' y, por el contrario, podrán ofrecerse para ese sujeto, en esas condiciones, buenas razones para aceptar que $p$ es un hecho.

Por consiguiente, en mi opinión, la adecuada elucidación de la noción de verdad es la siguiente:

' $p$ ' es verdadera si y sólo si

i) $p$ es un hecho (contruido por todo marco conceptual donde ' $p$ ' tiene sentido y ha preservado su significado), $y$

ii) en todos los marcos conceptuales donde ' $p$ ' tiene sentido y ha preservado su significado, pueden encontrarse razones a favor de ' $p$ ' y ' $p$ ' no puede ser rebatida por razones dentro de esos marcos conceptuales. 
Por otra parte, sostener que ' $p$ ' es verdadera es

$\left.i^{\prime}\right)$ pretender que $p$ es un hecho, $y$

$\left.i i^{\prime}\right)$ pretender que podríamos convencer a cualquiera, en una discusión racional en condiciones óptimas, de que aceptara ' $p$ ', es decir, que aceptara que $p$ es un hecho.

Mi insistencia en una concepción epistémica de la verdad es en que ii) tiene que ser aceptado como parte de lo que quiere decir que una proposición ' $p$ ' sea verdadera, además de aceptar i), por supuesto. Pero esto no debe confundirse con lo que quiere decir sostener o pretender que la proposición ' $p$ ' es verdadera, lo cual significa comprometerse con $\mathrm{i}^{\prime}$ ) y con $\mathrm{ii}^{\prime}$ ). Esto podrá parecer trivial a muchas personas, pero me parece que en ocasiones ha habido mucha confusión acerca de estas ideas en el contexto de la discusión alrededor de la concepción epistémica de la verdad.

\section{Verdad y justificación, realidad e intersubjetividad}

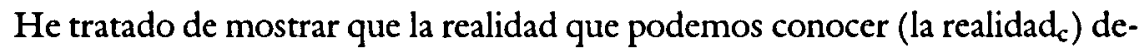
pende de la realidad independiente $y$ de los marcos conceptuales. Al depender también de los marcos conceptuales, la realidad ${ }_{c}$ es construida intersubjetivamente, de la misma manera que los marcos conceptuales son construidos, sostenidos y transformados intersubjetivamente. Entonces, los conceptos de "realidad ${ }_{c}$ " y de "intersubjetividad" están emparentados.

Pero el concepto de "verdad" tampoco está desligado del de "justificación". En primer lugar, hemos visto que desde la perspectiva internalista la verdad es, por un lado, adecuación de las proposiciones o de las creencias a la realidad. Pero se trata de la realidad. . Yo no puedo pretender que un enunciado es verdadero, sin pretender al mismo tiempo que lo que enuncia es. Pero pretender que lo que enuncia es, es pretender que ha sido construido a partir de la realidad independiente y por la acción de mi marco conceptual, y que por

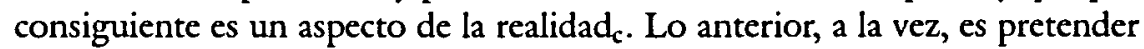
que existe plena justificación racional dentro de mi marco para aceptar, tanto que ' $p$ ' es verdadera, como que $p$ es un hecho, y que no puede existir razón alguna en mi marco que me convenza de que el hecho enunciado no es.

Como subrayé arriba, la pretensión de la que se habla no es una pretensión de la que tenga que estar consciente un sujeto epistémico al sostener la verdad de una proposición ' $p$ '. Al sostener la verdad de ' $p$ ', un sujeto epistémico pretende que $p$ es, y esto implica que existen razones a favor de aceptar ' $p$ ', y que no pueden existir buenas razones en su contra. Pero de esto último no tiene que estar consciente el sujeto que sostiene la verdad de ' $p$ '. 
Creo, pues, que es cierto que "verdad" y "justificación" responden a preguntas diferentes, pero también creo que son preguntas indisolublemente ligadas.

La conexión entre verdad y justificación puede apreciarse mejor si examinamos la siguiente proposición:

V) “' $p$ ' es verdadera aunque no sea aceptable por todo sujeto en condiciones ideales".

Desde el punto de vista que defiendo, contra una opinión generalizada, V) es contradictoria.

Para ver porqué, es necesario reiterar un razonamiento que ya he sugerido: si ' $p$ ' es verdadera, entonces $p$ es un hecho, pero eso significa que $p$ es construido por mi marco conceptual, por consiguiente, desde la perspectiva de mi marco conceptual y en condiciones epistémicas óptimas y en condiciones ideales de diálogo, nadie puede rechazar racionalmente que $p$. Es decir, todo sujeto en condiciones epistémicas óptimas y en una situación ideal de diálogo, admitiría que ' $p$ ' es verdadera.

Comprender qué significa que una creencia o una proposición sea verdade$r a$, es diferente a comprender qué pretendemos al afirmar que una proposición o que una creencia es verdadera. Hemos visto que se trata, en efecto, de dos cosas distintas. Pero en la primera se implica algo sobre la realidad $y$ algo sobre la aceptabilidad racional, y en la segunda se pretende también algo en relación con la realidad y algo también con respecto a la aceptabilidad racional de la proposición. He sugerido que en ambos casos está involucrada la realidad y la aceptabilidad racional porque los hechos y los objetos que realmente existen dependen de los marcos conceptuales, y éstos a su vez sólo existen en virtud de nuestras capacidades racionales.

\section{Conclusión}

Desde el punto de vista que he defendido, la verdad es una característica de las proposiciones que no puede perderse. Por consiguiente, la verdad no es relativa a cada marco conceptual, en el sentido de que ' $p$ ' pudiera ser verdadera en un marco conceptual, digamos $M C_{1}$, y falsa en otro marco distinto, digamos $\mathrm{MC}_{2}$. Pero la versión de realismo que defiendo admite que el mundo de $M C_{1}$ puede no ser el mismo de $\mathrm{MC}_{2}$, en un sentido ontológico fuerte. Esto es, puede haber hechos u objetos en $\mathrm{MC}_{1}$ que no existen ni pueden existir en $\mathrm{MC}_{2}$ (mientras que $\mathrm{MC}_{2}$ mantenga su identidad y no se trate de un marco conceptual construido a partir de $\mathrm{MC}_{2}$ pero que ya no es el mismo). Pero si un enunciado ' $p$ ' es verdadero en $M C_{1}$, y también puede expresarse con sentido dentro de $\mathrm{MC}_{2}$, preservando su significado, entonces ' $p$ ' tiene 
que ser verdadero en $\mathrm{MC}_{2}$, lo sepan o no los usuarios de este último marco conceptual.

Hemos visto, pues, un esbozo de cómo desarrollar una concepción pragmatista o internalista -en la línea sugerida por Putnam - que sea compatible a la vez con un relativismo conceptual y ontológico y con una posición realista, y una concepción no relativista de la verdad, según la cual ésta es vista como adecuación y al mismo tiempo como aceptabilidad racional en condiciones epistémicas y de diálogo óptimas.

\section{BibLiografía}

Putnam, H., 1981, Reason, Truth and History, Cambridge University Press.

- 1987, The Many Faces of Realism, Open Court, Lasalle (III.).

- 1990, Realism with a Human Face, Harvard University Press.

Villoro, L., 1990, "Sobre justificación y verdad: respuesta a León Olivé", Crítica (México), vol. XXII, no. 65, 1990, pp. 73-92. 